Int. J. Odontostomat.,

7(3):389-394, 2013.

\title{
Estado de Salud Oral y Asistencia al Control Odontológico en Escolares de 12 Años, Comuna de Penco, Región del Biobío
}

\author{
Oral Health Status and Dental Assistance Control in 12-year-old \\ School Children in the Penco Municipality, Biobío Region
}

María Jesús Gaete Forno*; Camila Córdova Cisterna* \& Patricio Oliva Mella*

GAETE, F. M. J.; CÓRDOVA, C. C. \& OLIVA, M. P. Estado de salud oral y asistencia al control odontológico en escolares de 12 años, comuna de Penco, Región del Biobío. Int. J. Odontostomat., 7(3):389-394, 2013.

RESUMEN: La caries dental y la enfermedad periodontal poseen una alta prevalencia en los distintos grupos etarios a nivel nacional generando un problema sanitario complejo. El objetivo fue evaluar el estado de salud oral y asistencia al control odontológico en pacientes escolares de 12 años en la comuna de Penco. Se realizó un estudio observacional analítico con un muestreo sistemático de 214 de escolares de 12 años. La prevalencia de caries fue de 61,03\%, sin diferencia estadística entre sexo (valor p 0,29). El COPD promedio fue 3,44. El 47\% asistió al dentista en los últimos 6 meses. El promedio COPD disminuyó en los últimos 10 años (de 4,77 a 3,44), siendo estadísticamente significativo (valor $\mathrm{p} \sim 0,00)$. De acuerdo a los indicadores observados, se concluye que los escolares de 12 años presentan un mal estado de salud oral y menos de la mitad ha asistido a control los últimos 6 meses.

PALABRAS CLAVE: salud oral infantil, salud oral en escolares, controles odontológicos infantiles.

\section{INTRODUCCIÓN}

Recomendaciones internacionales indican una evaluación continua de la salud oral en la población entre 5 y 7 años, sin embargo, se ha establecido los 12 años como la edad global de vigilancia de la caries y medición oficial de la enfermedad según la OMS (Petersen, 2003).

En Chile, la caries dental y enfermedad periodontal poseen una alta prevalencia en la población escolar (Sheiham, 2005), hace una década un $86 \%$ de los escolares de 12 años presentaban caries, con un Índice COPD de 3,42 en los dientes definitivos (Urbina et al., 1999), lo que implica un gasto de recursos y en tratamientos que podrían evitarse si se aumentan las medidas de prevención (Palomer, 2006). Es por ello que los estudios epidemiológicos de salud oral en escolares constituyen la base en la planificación de los programas de prevención y de asistencia dental (Rubio et al., 1997).
El diagnóstico de salud oral posee un índice de historia de caries en los escolares de 12 años de 1,9 en la dentición definitiva (Soto et al., 2007). Fernández et al. (2011) evaluaron los escolares de 12 años de establecimientos rurales y urbanos de la Región del Maule, donde $63,4 \%$ presentaban caries y un índice de daño de caries promedio de 3,15.

Las medias varían al desagregarse en ciudades, por ejemplo en la ciudad de Penco, el último estudio que se realizó para evaluar el estado de salud oral de los escolares de 12 años de la población fue hace 10 años, el cual se obtuvo un COPD de 4,77 (Torres \& Utrera, 2002), algo superior al promedio nacional.

Ante tal escenario, el Ministerio de Salud de Chile, ha establecido dentro de sus Objetivos Sanitarios de Salud Oral, 2000-2010 disminuir la caries en población 
menor de 20 años y aumentar el acceso a la atención odontológica a un $50 \%$, en los menores de 20 años (Ministerio de Salud, 2002). Para cumplir con aquello es necesario desarrollar programas de promoción y prevención de salud bucodental enfocados en dicha población previo conocimiento del estado real y actual de dicha población a nivel global y desagregado en comunas.

Por lo cual se establece como objetivo primordial del presente estudio la evaluación del estado de salud oral y control odontológico en escolares de 12 años de la Comuna de Penco, Concepción, 2012.

\section{MATERIALE Y MÉTODO}

Se diseño un estudio observacional analítico. El universo del estudio fueron 480 estudiantes de 12 años de la ciudad de Penco, se utilizó un muestreo probabilístico sistemático con 214 pacientes. Se establece un protocolo de reclutamiento de la muestra de forma proporcional manteniendo una relación equivalente entre la cantidad de alumnos por curso (Tabla I). Criterios de selección

a) Los alumnos escogidos debían cursar sexto o séptimo básico en el año 2012, b) haber nacido el año 2000 (se incorporaron los niños nacidos el año 1999 que aun no cumplían los 13 años).
Procedimiento clínico. Se realizó análisis clínico en salas acondicionadas, bajo luz natural y bandejas de examen completa y estéril (espejo $n^{\circ} 5$, sonda curva y pinza), guantes, mascarilla, linterna tipo cintillo y algodón.

Los datos obtenidos fueron registrados en una ficha clínica, realizada bajo los criterios de la OMS para Estudios Básicos de Salud Oral (OMS, 1987) y adaptada de acuerdo a los objetivos del estudio. Se evaluó el estado de los dientes y su necesidad de tratamiento mediante un odontograma, el Índice de Higiene Oral Simplificado (IHOS) (Green \& Vermillion, 1964), Índice de maloclusión (OMS, 1997). Se determinó el Índice COPD propuesto por Klein et al. (1938) y el Índice de Significancia de Caries (isC) (Bratthall, 2000). El IsC fue introducido el año 2000 por Bratthall, ya que existía una desigualdad en la distribución de la caries dental en una población dada, pues existen valores extremos que influyen en el promedio COPD lo que puede llevar a conclusiones erróneas (Fernández et al.).

Para la calibración de los evaluadores se realizó mediante una medición preliminar de 10 pacientes calculando una concordancia de $95 \%$.

Procedimiento cuantitativo. Se realizó una encuesta dirigidas a los adolescentes para la evaluación de la asistencia del control odontológico. Los ítems fueron:

Tabla I. Distribución de los escolares de 12 años por establecimiento educacional.

\begin{tabular}{lccc}
\hline Establecimiento Educacional & n Total & n Muestra & \% \\
\hline Tipo de Escuela & & & \\
$\quad$ Municipal & 233 & 105 & 49,3 \\
$\quad$ Subvencionado & 247 & 108 & 50,7 \\
Total & 480 & 213 & 100 \\
Escuelas Municipales & & & \\
$\quad$ República Italia & 55 & 20 & 9,4 \\
$\quad$ Escuela de Penco & 34 & 14 & 6,6 \\
$\quad$ Isla de Pascua & 84 & 45 & 21,1 \\
$\quad$ Los Conquistadores & 60 & 26 & 12,2 \\
Escuela Particular Subvencionada & & & \\
$\quad$ Queen Elizabeth & 40 & 17 & 8 \\
$\quad$ C. Educacional Gloria Mendez & 71 & 34 & 16 \\
$\quad$ El Refugio & 69 & 43 & 20,2 \\
$\quad$ Funny School & 67 & 14 & 6,6 \\
Total $\quad$ & 480 & 213 & 100 \\
\hline
\end{tabular}


- Tipo de de previsión (Fonasa, Isapre, Fuerza armada, PRAIS),

- Última visita al dentista

- Motivo de consulta.

Análisis estadístico. Los datos se generaron en una planilla del programa SPSS $17 \AA$, para el análisis descriptivo se calculó: media, mediana y las medidas de dispersión (mínimo, máximo, desviación estándar). Para el análisis inferencial se estableció un criterio de significancia estadística $p<0,05$; Evaluación de la normalidad se aplicó la prueba de Kolmogorov Smirnov para las escuelas que tienen más de 30 alumnos y de Shapiro Wilk para las escuelas que tiene menos de 30 alumnos. Para la comparación de medias, se utilizaron las dócimas de Kruskal-Wallis y Prueba T para una muestra. Para la comparación múltiple se utilizó la Prueba de Dunn y la asociación de variables cualitativas se evaluó con la prueba Chi- Cuadrado.

Aspectos bioéticos. Se elaboró una carta dirigida al Director solicitando autorización a las instituciones para realizar el estudio. Una vez otorgado el permiso, se envió los padres un consentimiento informado explicando el propósito del estudio, procedimiento y beneficio del presente estudio, realizado según las normas de Helsinki. El estudio fue aprobado por el Comité Científico de la Facultad de Odontología de la Universidad del Desarrollo.

Tabla II. Control Asistencia Odontológica en escolares de 12 años, Penco, Chile, 2012.

\begin{tabular}{|c|c|c|}
\hline Preguntas & $\mathbf{n}$ & $\%$ \\
\hline \multicolumn{3}{|l|}{ Previsión } \\
\hline Fonasa & 148 & 69,5 \\
\hline Otro ( Isapre, fuerza armada, PRAIS) & 63 & 29,6 \\
\hline No lo recuerdo & 2 & 0,9 \\
\hline Total & 213 & 100 \\
\hline \multicolumn{3}{|l|}{ Ultima visita al dentista } \\
\hline Menos de 6 meses & 101 & 47,4 \\
\hline Entre 6 y 12 años & 40 & 18,8 \\
\hline Más de un año & 49 & 23 \\
\hline No lo recuerdo & 16 & 7,5 \\
\hline Nunca he ido & 7 & 3,3 \\
\hline Total & 213 & 100 \\
\hline \multicolumn{3}{|l|}{ Motivo de Consulta } \\
\hline Control & 44 & 20,7 \\
\hline Tratamiento & 129 & 60,6 \\
\hline Dolor & 5 & 2,3 \\
\hline Extracción & 21 & 9,9 \\
\hline No lo recuerdo & 7 & 3,3 \\
\hline Nunca he ido & 7 & 3,3 \\
\hline Total & 213 & 100 \\
\hline
\end{tabular}

\section{RESULTADOS}

De los 214 escolares escogidos de los diferentes establecimientos educacionales de la comuna de Penco, participaron 213 pacientes $(99,5 \%)$. Su distribución por sexo fue similar, un $50,2 \%$ fueron mujeres y $49,8 \%$ hombres (del $99,5 \%$ ).

La prevalencia de los individuos libres de caries es de $38,96 \%$, de los cuales un $39,75 \%$ no presenta historia de caries y un 60,24 se encuentra sano pero tratado. En la prevalencia de caries entre sexo de los escolares, no se encontró diferencia estadística (valor $p \sim 0,29$ ).

La previsión de los consultados se remite principalmente a Fonasa $(69,5 \%)$, siendo la última visita hace menos de 6 meses y cuyo motivo de la consulta fue continuar con un tratamiento odontológico (Tabla II).

El Índice COPD promedio de los adolescentes fue de 3,44 $\pm 2,67$ (DE). Respecto al promedios de cada componente, el componente caries correspondió $1,79 \pm 2,2$ (DE), obturadas 1,54 $\pm 1,78$ (DE) y perdidas $0,11 \pm 0,45$ (DE) (Tabla III). El Índice CEO promedio encontrado en los individuos es de 0,59. El colegio con menor presencia de caries fue la escuela

Queen Elizabeth y el de mayor, escuela Isla de Pascua. Al comparar el promedio COPD de cada colegio, utilizando la prueba Kruskal-Wallis, se encontró diferencia estadística $(p \sim 0,01)$. Posteriormente según la Prueba de Dunn se observó que la diferencia se produce entre el colegio Los Conquistadores e Isla de Pascua. El IsC obtenido en los escolares de 12 años de la comuna de penco es de $6,36 \pm 1,83$ (DE).

Respecto al higiene oral, se utilizó el IHOS, el cual se obtuvo un promedio de $1,26 \pm 0,67$ (DE). Un $43,2 \%$ de los escolares presenta un higiene oral óptimo, un $44,1 \%$ un higiene oral regular y un $12,7 \%$ un higiene oral malo. La escuela con mayor higiene óptimo fue República de Italia y el menor higiene óptimo Isla de Pascua. Al comparar el promedio del IHOS por escuela, se encontró diferencia estadística (valor p 0,00) (Tabla IV). Se- 
gún lo obtenido por la Prueba de Dunn, la escuela Isla de Pascua tiene un IHOS estadísticamente distinto a las escuelas República de Italia, Queen Elizabeth y Los conquistadores. También se encontró diferencia estadística entre la Escuela República y Complejo Educacional Gloria Mendez.
Para el Índice de Maloclusión, un 60,1\% tuvo algún grado de anomalía dentomaxilar, donde $23,9 \%$ es leve y $36,2 \%$ moderado a severo. Respecto a la necesidad inmediata de tratamiento, el $8 \%$ necesita atención, $99 \%$ por causa de infección (extracción o necesidad de endodoncia) (Tabla V).

Tabla III. Índice COPD en escolares 12 años, según escuelas de Penco, 2012.

\begin{tabular}{lcccc}
\hline \multirow{2}{*}{ Escuela } & $\mathbf{C}$ & $\mathbf{O}$ & $\mathbf{P}$ & COPD \\
\cline { 2 - 5 } & Media $\pm \mathrm{DE}$ & Media $\pm \mathrm{DE}$ & Media $\pm \mathrm{DE}$ & Media \pm DE \\
\hline República & $1,35 \pm 1,72$ & $1,10 \pm 1,33$ & $0,25 \pm 0,91$ & $2,6 \pm 2,16$ \\
E. Penco & $1,5 \pm 1,69$ & $2,71 \pm 2,05$ & $0,74 \pm 0,26$ & $4,14 \pm 2,28$ \\
El Refugio & $1,9 \pm 2,50$ & $1,11 \pm 1,29$ & 0 & $3,0 \pm 2,6$ \\
Queen Elizabeth & $0,52 \pm 1$ & $2,52 \pm 1,84$ & 0 & $3,05 \pm 2,27$ \\
C. E. Gloria Mendez & $2,11 \pm 2,26$ & $1,02 \pm 1,62$ & $0,23 \pm 0,55$ & $3,38 \pm 2,66$ \\
Isla de Pascua & $2,91 \pm 2,54$ & $1,68 \pm 2,06$ & $0,24 \pm 0,57$ & $4,8 \pm 3,20$ \\
Conquistadores & $0,80 \pm 1,13$ & $1,53 \pm 1,65$ & 0 & $2,34 \pm 1,97$ \\
Funny School & $1,42 \pm 1,98$ & $1,92 \pm 2,16$ & 0 & $3,42 \pm 2,17$ \\
Total & $1,79 \pm 2,2$ & $1,54 \pm 1,78$ & $0,11 \pm 0,45$ & $3,44 \pm 2,67$ \\
\hline
\end{tabular}

Tabla IV. Índice de Higiene Oral Simplificado según escuelas, Penco 2012.

\begin{tabular}{lccccccc}
\hline \multirow{2}{*}{ Escuela } & \multicolumn{3}{c}{ Optimo } & \multicolumn{3}{c}{ Regular } & \multicolumn{2}{c}{ Malo } & IHOS \\
\cline { 2 - 9 } & $\mathbf{n}$ & $\mathbf{\%}$ & $\mathbf{n}$ & $\mathbf{\%}$ & $\mathbf{n}$ & $\mathbf{\%}$ & Media \pm DE \\
\hline República & 15 & 75 & 5 & 25 & --- & -- & $0,77 \pm 0,45$ \\
E. Penco & 6 & 42,9 & 7 & 50 & 1 & 7,1 & $1,3 \pm 0,49$ \\
El Refugio & 18 & 41,9 & 19 & 44,2 & 6 & 14 & $1,16 \pm 0,66$ \\
Queen Elizabeth & 12 & 70,6 & 5 & 29,4 & ---- & --- & $0,95 \pm 0,46$ \\
C. E. Gloria Mendez & 12 & 35,3 & 15 & 44,1 & 7 & 20,6 & $1,5 \pm 0,63$ \\
Isla de Pascua & 11 & 24,4 & 23 & 51,1 & 11 & 24,4 & $1,66 \pm 0,73$ \\
Conquistadores & 14 & 53,8 & 10 & 38,5 & 2 & 7,7 & $0,99 \pm 0,67$ \\
Funny School & 4 & 28,6 & 10 & 71,4 & ----- & --- & 1,3 \\
Total & 92 & 43,2 & 94 & 44,1 & 27 & 12,7 & $1,26 \pm 0,67$ \\
\hline
\end{tabular}

Tabla V. Malaoclusiones según escuelas Penco, 2012.

\begin{tabular}{lcccccc}
\hline \multirow{2}{*}{ Escuela } & \multicolumn{2}{c}{ Normal } & \multicolumn{2}{c}{ Leve } & \multicolumn{2}{c}{ Moderado - Severo } \\
\cline { 2 - 7 } & $\mathbf{n}^{\circ}$ & $\mathbf{\%}$ & $\mathbf{n}^{\circ}$ & $\%$ & $\mathbf{n}^{\circ}$ & $\%$ \\
\hline República & 8 & 40 & 8 & 40 & 4 & 20 \\
E. Penco & 5 & 35,7 & 3 & 21,4 & 6 & 42,9 \\
El Refugio & 16 & 37,2 & 10 & 23,3 & 17 & 39,5 \\
Queen Elizabeth & 3 & 17,6 & 6 & 35,3 & 8 & 47,1 \\
C. E. Gloria Mendez & 17 & 50 & 5 & 14,7 & 12 & 35,3 \\
Isla de Pascua & 18 & 40 & 8 & 17,8 & 19 & 42,2 \\
Conquistadores & 10 & 38,5 & 8 & 30,8 & 8 & 30,8 \\
Funny School & 8 & 57,1 & 3 & 21,4 & 3 & 21,4 \\
Total & 85 & 39,9 & 51 & 23,9 & 77 & 36,2 \\
\hline
\end{tabular}




\section{DISCUSIÓN}

La prevalencia de caries en la comuna de Penco (analizados 213 escolares de 12 años) resultó alta con $61,03 \%$, pero al compararse con otros estudios realizados a nivel nacional, se puede observar que es similar al estudio realizado por Soto et al. el año 2007 con un $62,5 \%$, pero inferior a los datos reportados por Urbina et al. (1996) con un $81,1 \%$. En relación a la historia de caries, se determinó que el $84,5 \%$ fue inferior al $97 \%$ obtenido el año 2002 en la comuna de Penco (Torres \& Utrera).

Por otro lado, al comparar el promedio COPD con el mismo estudio citado, se observó una disminución del 4,77 al 3,44. A pesar de la reducción del valor de este índice, la OPS recomienda que este valor sea inferior a 3 en los niños de 12 años, por lo que se considera de igual manera alterado.

Respecto a los componentes del COPD del mismo estudio demostró una disminución de caries y perdidos. El componente caries $(\mathrm{C})$ disminuyó de un promedio de 3,75 a un 1,79 , del perdido $(P)$ era de un 0,17 a un 0,11 . Otros componentes de la variable, como obturado (O) aumentó desde un 0,85 a 1,54. Estos cambios se relacionan con la incorporación del programa de Salud oral integral para niños y niñas de 6 años el año 2006 y del Programa JUNAEB móvil lo que abarca una mayor cobertura en los tratamientos dentales. Los valores sin embargo, son elevados, lo cual no cumple con la meta plateada por el Ministerio de Salud, alcanzar un promedio COPD 1,9 el año 2010. El índice del estudio se aleja del obtenido el año 2007 por Soto et al. con un COPD 1,9, lo cual concluyó que Chile cumplió este objetivo sanitario.

Al analizar el promedio del IHOS obtenido con el estudio realizado hace 10 años en la comuna, se observa un aumento de 0,54 a 1,26 (valor p 0,00). Al comprar los resultados obtenidos con el estudio realizado por Fernández et al. en la Región del Maule se observó que el promedio COPD obtenido es levemente mayor (Penco 3,44; Región del Maule 3,15), al igual que el IsC (Penco: 6,36 \pm 1,83 (DE), Región Maule área urbana: 5,24 \pm 1,52 (DE)). Nishi et al. (2002), establecieron en su estudio que valor del Is $C$ debe ser inferior a 3, en los escolares de 12 años para el año 2015. El IsC se calcula de la siguiente manera: los individuos se ordenan en función de sus valores CPOD, a continuación, la tercera parte de la población con el puntaje más alto se selecciona y se calcula el CPOD promedio para este subgrupo (Bratthall).

Respecto a los resultados obtenidos sobre el control odontológico se estableció que el $66,2 \%$ ha asistido al dentista, similar al obtenido en el estudio de Soto et al. (un $64,2 \%$ ). Se observó que se cumple el objetivo sanitario plateado por el Ministerio de Salud de alcanzar una cobertura de $50 \%$ en la atención odontológica en los menos de 20 años.

Aún existe un deficiente estado de salud oral de los escolares de 12 años, no cumpliendo con la totalidad de los objetivos sanitarios plateados por el Ministerio de Salud de Chile. Esto se transforma en un problema sanitario, dado que una mala salud bucodental puede tener profundas repercusiones en la salud general y en la calidad de vida (Petersen), por lo cual se hace necesario implementar nuevos programas orientados a la promoción, prevención y educación en salud oral en comuna de Penco.

\section{CONCLUSIÓN}

De acuerdo al estudio realizado, los escolares de 12 años presentan un mal estado de salud oral y más de la mitad de los adolescentes han asistido al control odontológico el último año.

GAETE, F. M. J.; CÓRDOVA, C. C. \& OLIVA, M. P. Oral Health Status and Dental Assistance Control in 12-year-old School Children in the Penco Municipality, Biobío Region. Int. J. Odontostomat., 7(3):389-394, 2013.

ABSTRACT: Dental caries and periodontal disease have a high prevalence in the different age groups, creating a complex health problem. The objective of this study was to evaluate the state of oral health and dental attendance controls in 12-year-old patients at the elementary school in the town of Penco. We used a descriptive trans-sectional study with systematic sample of two hundred and fourteen (214) 12-year-old schoolchildren. The results showed a prevalence of caries of $61.03 \%$, with no statistical difference between sexes (value $p \sim 0.29$ ). The COPD average was 3.44 . Of the studied population $47 \%$ have been attended by the dentist in the last 6 months. The average COPD has declined in the last 10 years (from 4.77 to 3.44 ) with statistically significant value $p \sim 0.00$. According to the indicators observed, we conclude that 12 -year-old school children have poor oral health and less than half have been monitored in the last 6 months.

KEY WORDS: child oral health, oral health in school children, childhood dental controls. 


\section{REFERENCIAS BIBLIOGRÁFICAS}

Bratthall, D. Introducing the Significant Caries Index together with a proposal for a new global oral health goal for 12year-olds. Int. Dent. J., 50(6):378-84, 2000.

Fernández, G. C.; Núñez, F. L. \& Díaz, S. N. Determinantes de salud oral en población de 12 años Rev. Clin. Periodoncia Implantol. Rehabil. Oral, 4(3):117-21, 2011.

Green, J. C. \& Vermillion, J. R. The simplified oral hygiene index. J. Am. Dent. Assoc., 68:7-13, 1964.

Klein, H.; Palmer, C. E. \& Knutson, J. W. Studies on Dental Caries. I. Dental status and Dental Needs of elementary school chilidren. Public Health Rep., 53(19):751-65, 1938.

Ministerio de Salud. Objetivos Sanitarios para la década 2000-2010. El Vigía, 5(15):1-12, 2002.

Nishi, M.; Stjernswärd, J.; Carlsson, P. \& Bratthall, D. Caries experience of some countries and areas expressed by the Significant Caries Index. Community Dent. Oral Epidemiol., 30(4):296-301, 2002.

Organización Mundial de la Salud (OMS). Encuestas de Salud Bucodental. Métodos Básicos. $4^{a}$ ed. Ginebra, Organización Mundial de la Salud, 1997.

Organización Mundial de la Salud (OMS). Oral health surveys. Basic Methods. $3^{\text {rd }}$ ed. Geneve, World Health Organization, 1987.

Palomer, L. Caries dental en el niño. Una enfermedad contagiosa. Rev. Chil. Pediatr., 77(1):56-60, 2006.

Petersen, P. E. The World Oral Health Report 2003: continuous improvement of oral health in the 21st century--the approach of the WHO Global Oral Health Programme. Community Dent. Oral Epidemiol., 31(Suppl. 1):3-23, 2003. En Texto Organización Mundial de la Salud

Rubio, C. J. M.; Robledo de Dios, T.; Llodra, C. J. C.; Simón, S. F.; Artazcoz, O. J.; González, A. V. L. \& García-Camba de la Muela, J. M. Criterios minimos de los estudios epidemiológicos de salud dental en escolares. Rev. Esp. Salud Pública, 71(3):231-42, 1997.

Sheiham, A. Oral health, general health and quality of life. Bull. World Health Organ., 83(9):644-5, 2005.

Soto, L.; Tapia, R.; Jara, G. \& Rodríguez, G. Diagnóstico de salud bucal de adolescente de 12 años y evaluación del grado de cumplimiento de los objetivos sanitarios de salud bucal 2000-2010. Santiago, Ediciones Universidad Mayor, 2007.
Torres, P. \& Utrera, C. Estado salud bucal de los escolares básicos de 12 años de la comuna de Penco, año 2002. Informe internado asistencial Cesfam Penco. Facultad Odontología, Universidad de Concepción, Chile, 2002.

Urbina, T.; Caro, J. C. \& Vicent, M. Caries dentaria y fluorosis en niños de 6, 8 y 12 años de la II, VI, VIII, IX, X y RM. Chile 1996. Santiago, División Programas de Salud, Departamento Odontológico MINSAL, 1996.

Urbina, T.; Caro, J. P. \& Vicent, M. Caries y fluorosis en niños de 6 a 8 años y 12 años. Santiago de Chile, Departamento Odontológico, Ministerio de Salud, 1999.

Dirección para Correspondencia:

María Jesús Gaete Forno

Facultad de Odontología

Universidad del Desarrollo Concepción

Barros Arana 1734, Concepción

CHILE

Fono: 041-2268544

Fax: $\quad 56-41-2268501$

Email: ma.jesusgaete@gmail.com

Recibido : 18-10-2012

Aceptado: 28-08-2013 